\title{
Potential Causes of Paraplegia after Coronary Artery Bypass Grafting: A Literature Review
}

\author{
Zeyi Cheng* ${ }^{1}$ Jin $\mathrm{Xu}^{*},{ }^{2}$ Yingqiang Guo ${ }^{1}$ \\ ${ }^{1}$ Department of Cardiovascular Surgery, West China Hospital, Sichuan University, Chengdu, Sichuan, China; ${ }^{2}$ Department of Cardiology, \\ the first hospital of Lanzhou University, Lanzhou, Gansu, China
}

\section{ABSTRACT}

Paraplegia is an unpredictable neurologic complication after coronary artery bypass grafting (CABG) surgery. It is rare but fatal, and the mechanism still is unclear. We aimed to make a summary of the possible causes of paraplegia after CABG. Pubmed database was searched from January 1, 1978 to December 31, 2019, and 14 studies were finally included. Paraplegia after CABG is a multifactorial consequence, but spinal cord ischemia is the key pathological factor to postoperative paraplegia.

\section{INTRODUCTION}

Background: Paraplegia is an unpredictable neurologic complication that could occur after aortic coarctation surgery, thoracotomy (such as lobectomy and pneumonectomy), aortic dissection (AD), cardiac valve surgery, and cardiac transplantation and CABG. The occurrence of paraplegia after these operations is mainly caused by spinal cord ischemia (SCI). However, paraplegia after CABG is rare but fatal. In this review, we summarized the possible mechanism of paraplegia after CABG, reviewed the anatomy and physiology of the spinal cord, extracted the clinical manifestations and complications as well as the related treatments and outcomes to further understand the possible mechanisms so as to prevent paraplegia after CABG in our clinical work.

Anatomy and physiological function of the spinal cord: The spinal cord acts as a connection center between the central and peripheral nervous systems for sensory and motor function. It is supported and protected by various structures: encased in the vertebral column, the cord originates at the medulla and ceases at the second lumbar vertebra. Within the vertebral column, the meninges and cerebrospinal fluid (CSF) surround and protect the cord [Donohoe 2000]. The

Received February 22 2021; received in revised form April 10, 2021; accepted April 12, 2021.

*Zeyi Cheng and Fin Xu contributed equally.

Correspondence: Yingqiang Guo, Department of Cardiovascular Surgery, West China Hospital, Sichuan University, No. 37, Guoxue Alley, Wubou District, Chengdu, Sichuan 610041, China; +86-189-8060-1876 (e-mail: drguoyq@botmail.com). spinal cord has 31 segments (eight cervical, 12 thoracic or dorsal, five lumbar, five sacral, and one coccygeal), each of which (except the first cervical segment, which has only a ventral root) has a pair of dorsal and ventral roots and a pair of spinal nerves [Bican 2013].

The blood supply for the spinal cord mainly originates from the anterior spinal artery and posterior spinal artery as well as root artery. The anterior spinal arteries are large and supply the frontal two-thirds of the spinal cord, the posterior arteries perfuse the remaining cord. The junction of the two adjacent root arteries is a weak circulatory area. The artery of Adamkiewicz (AKA), also known as the great anterior radiculomedullary artery, joins the anterior spinal artery in the lower one-third of the spinal cord. Because of its large role in feeding the spinal cord, many studies have emphasized the importance of reattaching the intercostal or lumbar arteries to the AKA in the event of spinal cord ischemia, following vascular and endovascular surgery [Taterra 2019].

The possible causes of paraplegia after CABG: The incidence of paraplegia is rare but it is often irreversible, and the prognosis is poor. From the published studies, we concluded that paraplegia after CABG is a multifactorial consequence, such as using intra-aortic balloon pump (IABP), hypertension, microcirculation embolization, local anesthesia block or subdural hematomas, severe aortic calcification, spinal cord injury during endotracheal intubation, left internal mammary artery bypass grafting, aortic and CABG surgery, and even electrolyte disorder, as well as the drug toxic effects. The most similar thing is that paraplegia after CABG is almost associated with SCI.

\section{METHODS}

We searched the English literature through Pubmed database from January 1, 1978 to December 31, 2019. Search strategies were " $((()(()(()(()(()(((($ Paraplegia[MeSH Terms] $)$ OR (Paraplegias[Title/Abstract])) OR (Paralysis, Legs[Title/ Abstract])) OR (Paralysis, Lower Limbs[Title/Abstract])) OR (Paralysis, Lower Extremities[Title/Abstract])) OR (Paraplegia, Spinal[Title/Abstract])) OR (Paraplegias, Spinal[Title/Abstract])) OR (Spinal Paraplegia[Title/ Abstract])) OR (Spinal Paraplegias[Title/Abstract])) OR (Cerebral Paraplegia[Title/Abstract])) OR (Cerebral Paraplegias[Title/Abstract])) AND (Coronary Artery Bypass Grafting $[\mathrm{MeSH}])$ ) OR (Artery Bypass, Coronary[Title/ 
Case reports of paraplegia after CABG in the English literature

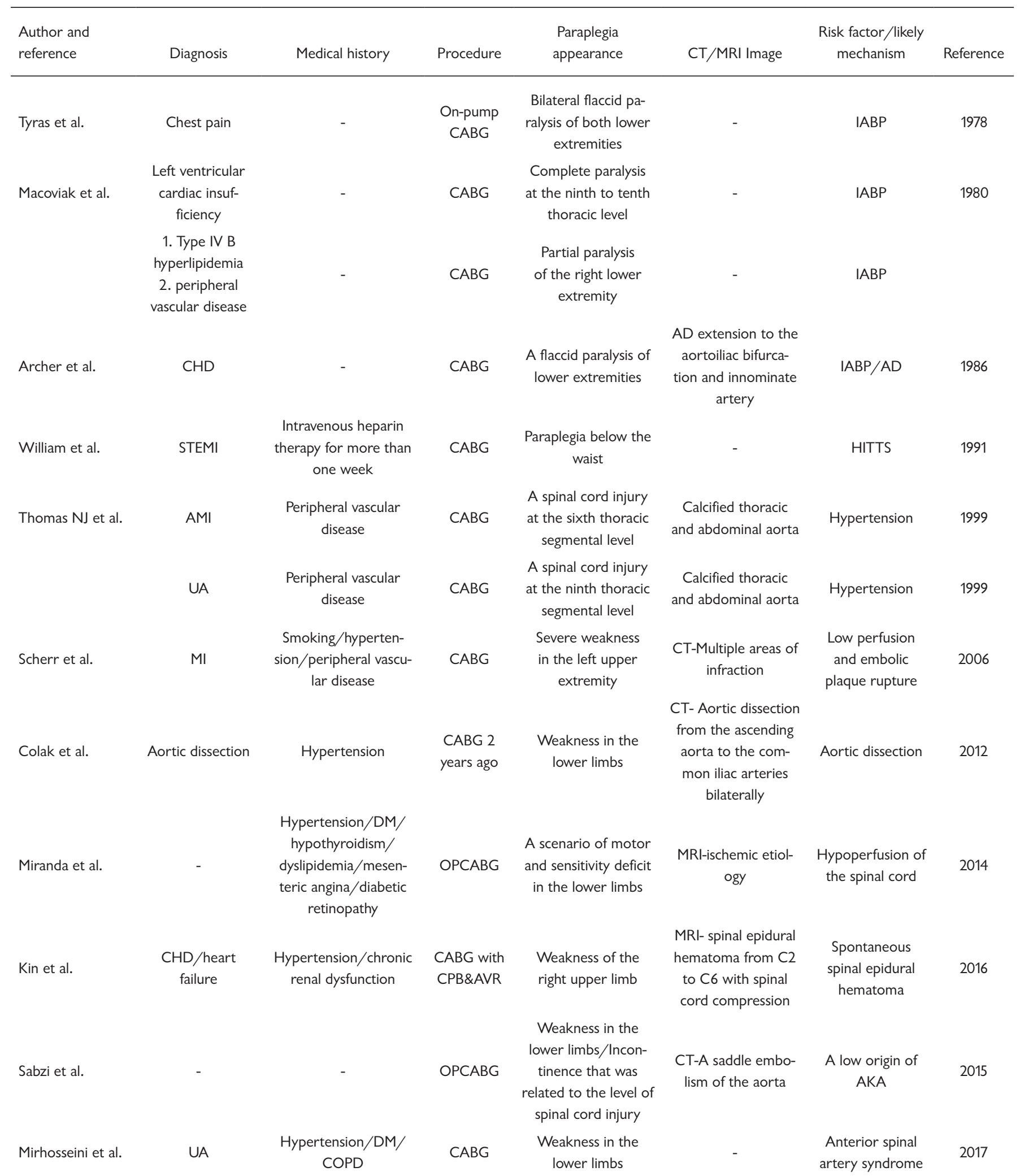


Case reports of paraplegia after CABG in the English literature

\begin{tabular}{|c|c|c|c|c|c|c|c|}
\hline Haidari et al. & Stable angina & $\begin{array}{l}\text { Hypertension/DM/ } \\
\text { CKD/peripheral vascu- } \\
\text { lar disease }\end{array}$ & OPCABG & $\begin{array}{l}\text { Areflexic paraplegia } \\
\text { associated with the } \\
\text { absence of sensation } \\
\text { in both lower ex- } \\
\text { tremities and muscle } \\
\text { weakness with } \\
\text { reduced reflexes }\end{array}$ & $\begin{array}{c}\text { Herniation of C3-4 } \\
\text { and C6-7 }\end{array}$ & $\begin{array}{c}\text { Traumatic in- } \\
\text { jury and ischemic } \\
\text { event }\end{array}$ & 2017 \\
\hline
\end{tabular}

\begin{abstract}
MI, myocardial infarction; AF, atrial fibrillation; UA, unstable angina; ACS, acute coronary syndrome; CHD, coronary heart disease; DM, diabetes mellitus; VSD, ventricular septal defect; LIMA, left internal mammary artery; LIMA, left internal mammary artery; LAD, left anterior descending branch; OPCABG, off-pump coronary artery bypass graft; AVR, aortic valve replacement; TEVAR: thoracic endovascular aorta repair
\end{abstract}

Abstract])) OR (Artery Bypasses, Coronary[Title/Abstract])) OR (Bypasses, Coronary Artery[Title/Abstract])) OR (Coronary Artery Bypasses[Title/Abstract])) OR (Coronary Artery Bypass Surgery[Title/Abstract])) OR (Bypass, Coronary Artery[Title/Abstract])) OR (Aortocoronary Bypass[Title/ Abstract])) OR (Aortocoronary Bypasses[Title/Abstract])) OR (Bypass, Aortocoronary[Title/Abstract])) OR (Bypasses, Aortocoronary[Title/Abstract])) OR (Bypass Surgery, Coronary Artery[Title/Abstract])." The literature obtained was carefully reviewed by the inclusion and exclusion criteria. All papers were limited to English language.

Selection criteria and data extraction: Papers must include paraplegia and CABG surgery simultaneously. The exclusion criteria were: 1) No direct report of the paraplegia in the studies; 2) Animal experiments. Data extraction was independently conducted by two authors (ZY C and J X) who used a standardized data collection Excel form. Differences were decided by the senior author (YQ G). The clinical characteristics have been summarized (Table 1).

\section{RESULTS}

IABP: IABP is widely used in cardiogenic shock (CS), severe myocardial infarction (MI), high-risk percutaneous coronary intervention and cardiac surgery [White 2015]. Tyras et al [Tyras 1978] reported the first case about paraplegia after CABG, which was closely related to IABP. The post-mortem examination demonstrated a sub-adventitial hematoma of the thoracic aorta from the $6 \mathrm{~cm}$ above the left subclavian artery and extended to $5 \mathrm{~cm}$ proximal to the celiac artery; the hematoma affected six pairs of intercostal arteries supply and lead to SCI. Macoviak et al [Macoviak 1980] reported 5-year follow-up complications after using IABP.
The rate of IABP-related spinal cord paralysis was $1.7 \%$. Emboli and partial aortic dissection were the most primary causes. Harris et al [Harris 1986] reported a 72-year-old man, who underwent $C A B G$ and atrial septum defect repair performance but suffered acute paraplegia after inserting IABP. The autopsies showed SCI caused by cholesterol embolism. In sum, IABP could cause aortic intima rupture and fragmentation of aortic atherosclerotic plaques, which finally resulted in SCI.

Anterior spinal artery syndrome (ASAS): ASAS is always a devastating spinal stroke that occurs when the anterior spinal artery or one of its main supplying anterior medullary arteries is occluded. Bilateral paresis and loss of pain and temperature sensation with preservation of proprioception and vibratory sensation below the lesion are the typical characteristics [Müller 2012]. Mirhosseini et al [Mirhosseini 2017] reported a 61-year-old patient, who underwent hemiplegia in both lower extremities (muscle strength grade was zero) on the first day after surgery. Computer tomography angiography (CTA) showed multiple atherosclerotic plaques in the aortic artery, but no embolism; this patient had a long history of diabetes mellitus (DM) and hypertension and peripheral arterial diseases (PAD). According to anatomical characteristics of spinal cord blood supply, the Adamkiewicz artery is a myeloid artery that enhances the blood supply for three longitudinal vessels, and it is an important blood supply source for the anterior spinal cord artery and lumbar segment. The authors deduced that the internal mammary artery (IMA), as a collateral supplier of the artery of Adamkiewicz, once used as a conduit in CABG, which probably caused SCI. Subramaniam et al [Subramaniam 2017] recorded an 81-year-old woman with severe aortic stenosis (AS) and coronary artery disease (CAD) who underwent aortic valve replacement (AVR) and CABG surgery; when she was returned to intensive care 
unit (ICU), severe hemodynamic instability and low cardiac output occurred. The next day, the physical examination of the nervous system showed the patient's pain and temperature sense did not exist in all lower limb muscle groups. Spine magnetic resonance imaging (MRI) showed abnormal signal from T6-T7, extended to the cone, the central spinal cord became dilated, the abnormal signal appeared, and ischemic lesions were found in the $2 / 3$ area of the ventral spinal cord, which indicated ASAS.

Aortic dissection (AD): Aortic artery intima tears may occur after aortic cross-clamping, aortic cannulation, and when suturing the graft to the aorta wall. Archer et al [Archer 1986] reported a patient who got paralysis of the lower extremities after CABG surgery, but the patient maintained a sense of touch and vibration, CT indicated that the dissection arose from an intimal tear at the suture line of a coronary artery bypass graft. The intimal tear may occur in the site of intervention or trauma, such as aortic clamping or aortic catheterization. What's more, if the aortic wall itself has lesions, the possibility of $\mathrm{AD}$ occurrence after $\mathrm{CABG}$ will increase. Colak et al [Colak 2012] reported an interesting case about painless $\mathrm{AD}$. The patient underwent CABG two years ago with a history of hypertension. He had motor deficits in the lower extremities. CT revealed $\mathrm{AD}$ from the ascending aorta to the common iliac arteries bilaterally. Although painless paraplegia in association with acute aortic dissection was rare, $\mathrm{AD}$ should be considered in the differential diagnosis of patients who present emergently with acute-onset paraplegia (whether painful or painless) and pulseless femoral arteries bilaterally.

Adamkiewicz artery syndrome: Miranda et al [Miranda 2014] reported a 31-year-old female patient with hypertension and insulin-dependent diabetes, chronic renal failure with hemodialysis for two years, and other comorbidities like hypothyroidism, dyslipidemia, mesenteric ischemia, and diabetic retinopathy. Her clinical manifestations were motor and sensitivity deficit in the lower limbs on the first post-operation day after CABG. Haidari et al [Haidari 2017] reported a case of postoperative paraplegia after off-pump CABG (op-CABG) because of multiple risk factors, such as $\mathrm{PAD}$, DM, hypertension, chronic renal failure, intraoperative hypotension, and thoracic disc protrusion. Sixty hours after the patient arrived to the ICU, neurological physical examination showed flaccid and areflexic paraplegia associated with the absence of sensation and muscle weakness in both lower extremities. The same CABG strategy was applied in those two cases that the left IMA was used as a graft conduit resulted in decreased perfusion of the AKA artery and the anterior spinal artery. The second case included posterior paraspinal ligament injury, which may result in the posterior herniation of the intervertebral disk and finally lead to the compression and ischemia of the spinal cord.

The inner diameter of the AKA artery is about $0.8 \sim 1.3 \mathrm{~mm}$, and the emitting position is mostly between T8 L3. Previous study found that the root arteries mainly exist at the level of C6, T9, and L2, and the weak points of the anastomosis of the two root arteries mostly appear near the T4 and L1 segments. Therefore, although there is an anastomosis between the arterial branches, it still is not enough to establish an effective collateral circulation during ischemia. As a very rare anatomical abnormality, AKA artery syndrome occurs at the L3 or L4-L5 level in $1.4 \%$ and $0.2 \%$ of patients [Sabzi 2015]. Sabzi et al [Sabzi 2015] reported a 57-year-old male eight days after uneventful off-pump coronary artery bypass (OPCAB) presented with severe lower extremity pain and sudden fecal and urinary incontinence, followed by the absence of pulsations in the lower limbs and paraplegia, during a 20-minute period. Angiography showed a saddle embolism in the aorta. Saddle emboli cannot block the abnormal origin of these high arteries, lead to paralysis, so low origin of AKA may be a reasonable explanation for the occurrence of paralysis and cauda equina symptoms.

Systemic hypertension: Thomas et al [Thomas 1999] reported paraplegia and spinal cord injury in two patients with severe PAD who had hypertensive crises after coronary revascularization. One patient was a 65 -year-old woman with $\mathrm{CAD}$, hypertension, $\mathrm{DM}$, and severe symptomatic aortoiliac occlusive disease; she underwent routine 3-vessel CABG using the LIMA to bypass the left anterior descending coronary artery. About 30 minutes later, her mean blood pressure (MBP) was $>100 \mathrm{mmHg}$ and extremely difficult to control before decannulation, however, she awoke from anesthesia with a spinal cord injury at the sixth thoracic segmental level and paraplegia. Another patient was a 59-year-old man with a history of intermittent claudication, bilateral superficial femoral artery occlusions, and lupus erythematosus, inactive for 15 years. He underwent four-vessel bypass using the LIMA for the left anterior descending coronary artery proceeded uneventfully. When he was returned to the ICU, the MBP was $>100 \mathrm{mmHg}$. MBP was recorded at between 100 and 110 $\mathrm{mmHg}$, during the subsequent 6-hour period. Paraplegia was observed for the first time two hours later, during an attempt to move the patient to a chair; his level of injury was identified at the ninth thoracic vertebra. CTA scan of the aorta showed nearly circumferential, but irregular, calcification, and no evidence of dissection. The authors deduced that SCI was related to postoperative hypertensive crises associated with atheromatous involvement of the thoracic aorta, the rupture of an atheromatous plaque, or the raising of localized intimal flap results to microscopic embolization of debris was the most reasonable explanation.

Spontaneous spinal epidural hematoma (SSEH): SSEH is a relatively rare disease, the spinal epidural space is occupied by lesions, the main manifestation is sudden neck or back pain that progresses toward paraparesis or quadriparesis, depending on the level of the lesion. Kin et al [Kin 2016] reported an 86-year-old woman, who underwent AVR and CABG surgery. On postoperative day 3, she developed sudden-onset neck pain followed by weakness in the right arm. Her symptoms worsened, and finally, she developed paraplegia. At $60 \mathrm{~h}$ after the first complaint, SSEH from C2 to C6 with spinal cord compression was diagnosed by MRI of the cervical region. They recommend when neurological abnormalities with neck or back pain are observed after thoracotomy, SSEH should be considered preferentially, even though this was a rare case. Besides, if SSEH is suspected, CT/MRI exam should be considered in time. 
Anterior cerebral artery infarction: Anterior cerebral artery (ACA) infarction is closely related to paraplegia. Garg et al [Garg 2014] reported pure motor paraplegia resulted from bilateral anterior cerebral artery infarction after CABG. The cerebrovascular system involving the internal carotid artery and the anterior cerebral artery is rare. Bilateral anterior cerebral artery infarction is usually due to spasms, complicating subarachnoid hemorrhage caused by anterior communicating artery aneurysm. Another cause is thrombosis of the proximal part of one ACA when the contralateral proximal branch is rudimental or absent. ACA infarction usually affects the paracentral part of the frontal lobe and motor neurons, the position distribution is mostly related to the lower limbs. Dyskinesia with characteristic foot distribution is the most common neurological sign of ACA stroke.

Heparin-induced thrombocytopenia (HIT): Heparininduced thrombocytopenia (HIT) is heparin's most clinically relevant non-hemorrhagic complication. It is an immune complication caused by antibodies directed to complexes containing heparin and an endogenous platelet protein, platelet factor 4 [Pouplard 1999]. Platelet activation in HIT is also accompanied by intense thrombin generation. The mechanisms underlying thrombin generation in HIT are not fully clarified. A 52-year-old patient with acute inferior myocardial infarction received tissue plasminogen activator ( $\mathrm{r}-\mathrm{tPA})$ followed by intravenous heparin therapy for more than a week. He received 6 units of platelet transfusions for a postoperative platelet count of $4,600 / \mathrm{pL}$ and because of mild diffuse wound bleeding. Immediately after the operation, he suffered from moderate hypertension and low urine output. The aortic incision showed a large white thrombus lying in the distal aorta with its proximal extension to the renal arterial orifices. He was paralyzed below his waist. Histologic examination indicated that composed predominantly of platelet aggregates. Tests for heparin-dependent platelet-aggregating antibodies showed the patient's sera to be significantly positive [Feng 1993]. The mechanism appears to involve heparinimmunoglobulin complexes that react with $\mathrm{Fc}$ receptors on platelets, leading to diffuse platelet activation and aggregation and finally thrombosis [Mehta 1991].

\section{DISCUSSION}

Paraplegia after CABG is rare but affects the prognosis. The diagnosis of newly happened paraplegia after surgery is full of difficulties and challenges mainly because the patient has not recovered after the operation and the use of sedative and analgesic drugs in ICU. Meanwhile, the pacing wires and metal implants in the patient's body restrict early MRI tests. Before the CABG procedure, the surgeon should comprehensively evaluate the patient's general condition, including cardiovascular risk factors, like smoking, alcohol abuse, history of hypertension, hyperlipidemia, DM, PAD, atrial fibrillation, renal dysfunction, hemodialysis, and so on. The specific mechanism of paraplegia after CABG is difficult to explain, but from what we've discussed above, the most important pathological processes involve spinal cord ischemia, so preventive measures should be taken seriously during the perioperative period, improving the treatment of basic diseases and avoiding various possible risk factors during surgery procedure, such as decreased cholesterol level. Controlling the MBP of $90 \mathrm{mmHg}$ is necessary for the spinal cord to maintain perfusion. Meanwhile, antiplatelet and thrombolysis are used in most centers to prevent SCI caused by vascular obstruction and embolism [Scherr 2006]. Also, for high-risk patients, CT, MRI, laboratory tests and electromyography exams may be helpful to evaluate denervation. CT can show the hematoma, distinguish the flow rate between the arterial lumen and the pseudo-lumen, measure the increased aorta size, calcification lesion, and the location of SCI. MRI is the most proper way to detect spinal cord abnormality and locate spinal cord infarction position [Heldner 2012].

According to Bondurant et al [Bondurant 1990], the MRI image classifications of SCI are defined as the following three categories: 1) Type I: a decreased signal activity compatible with acute bleeding was detected; 2) Type II: high signal intensity consistent with acute spinal cord ischemia and edema was observed; and 3) Type III: a mixed signal of a central hypo-intensity and a peripheral hyper-intensity compatible with acute confusion was detected.

IABP-related paraplegia after CABG is the most common reason by reviewing the literature. The mechanisms include iatrogenic $\mathrm{AD}$ or adventitial hematoma caused by IABP and the occlusion of small arterioles by cholesterol or atheromatous emboli [White 2015; Tyras 1978; Macoviak 1980; Harris 1986]. Therefore, intensive attention should be paid to the aortic manipulation during $\mathrm{CABG}$, clamping, releasing, cannulating, both of which potentially increase the atherosclerotic plaque instability and cause ischemic stroke. Moreover, when neurological abnormalities with neck or back pain were observed after open cardiac surgery, spontaneous spinal epidural hematoma must be considered as part of the differential diagnosis, even if it is a rare case, CT/MRI should be performed as soon as possible [Kin 2016].

It is obvious that to prevent paraplegia after CABG, a comprehensive assessment of the patient's physical condition must be performed, modifying the surgical techniques and preventing SCI. IMA is an important blood supply to the collaterals of the spinal cord. When a patient received TEVAR and other thoracic aortic surgery, the use of IMA as a CABG conduit should be careful. There also are many methods to ensure optimized spinal perfusion, including the addition of systemic corticosteroids, keeping appropriate blood pressure and promoting diuresis. Besides, cerebrospinal fluid drainage is an effective recovery way of neurological dysfunction [Coselli 2000].

Although the current literature reported the causes of paraplegia after $\mathrm{CABG}$, the best management and prevention of paraplegia after CABG has not yet reached a consensus. Further exploration still is needed.

\section{CONCLUSION}

Paraplegia is a rare complication after CABG surgery and the mechanism remains unclear, but it is a multifactorial 
consequence. Early diagnosis and prompt treatment are crucial for a positive prognosis. The prevention and treatment of paraplegia after CABG should run through the entire perioperative period: comprehensive assessment of the risk factors of paraplegia before CABG, careful operation during the surgery procedure, and recognition of the occurrence of paraplegia as soon as possible after CABG through physical examination, patient's symptoms and signs, and CT/MRI imaging examinations.

This literature review summarized the possible causes of paraplegia after CABG. Obviously, the most important pathological process in paraplegia is spinal cord ischemia. The etiological research and specific molecular mechanisms, as well as intervention time and treatment targets, should be further explored.

\section{ACKNOWLEDGEMENT}

Funding: This study was supported by the National Natural Science Foundation of China (No. 81970345).

\section{REFERENCES}

Archer AG, Choyke PL, Zeman RK, et al. 1986. Aortic dissection following coronary artery bypass surgery: diagnosis by CT. Cardiovasc Intervent Radiol. 9(3):142-5.

Bican O, Minagar A, Pruitt AA. 2013. The spinal cord: a review of functional neuroanatomy. Neurol Clin. 31(1):1-18.

Bondurant FJ, Cotler HB, Kulkarni MV, et al. 1990. Acute spinal cord injury: a study using physical examination and magnetic resonance imaging. Spine (Phila Pa 1976). 15:630-8.

Colak N, Nazli Y, Alpay MF, et al. 2012. Painless aortic dissection presenting as paraplegia. Tex Heart Inst J. 39(2):273-6.

Coselli JS, LeMaire SA, Schmittling ZC, Koksoy C. 2000. Cerebrospinal fluid drainage in thoracoabdominal aortic surgery. Semin Vasc Surg. 13(4):308-314.

Donohoe DR. 2000. Neurologic system: physiology, assessment, and intracranial hypertension. In: Pass CCRN 2nd ed. St. Louis, Mo: CV Mosby. 293-346.

Feng WC, Singh AK, Bert AA, et al. 1993. Perioperative paraplegia and multiorgan failure from heparin-induced thrombocytopenia. Ann Thorac Surg. 55(6): 1555-7.

Garg A, Bansal A, Bhuyan S, et al. 2014. Paraplegia during coronary artery bypass graft surgery caused by bilateral anterior cerebral artery territory infarction. Ann Transl Med. 2(5):49.

Haidari A, Sabzi F, Dabiri S. 2017. Paraplegia after Off-Pump Coronary Artery By-Pass Grafting. Acta Med Iran. 55(12):807-11.
Harris RE, Reimer KA, Crain BJ, et al. 1986. Spinal cord infarction following intraaortic balloon support. Ann Thorac Surg. 42(2):206-7.

Heldner MR, Arnold M, Nedeltchev K, et al. 2012. Vascular diseases of the spinal cord: A review. Curr Treat Options Neurol. 14(6):509-20.

Kin H, Mukaida M, Koizumi J, et al. 2016. Spontaneous spinal epidural hematoma presenting as paraplegia after cardiac surgery. Gen Thorac Cardiovasc Surg. 64(3):153-5.

Macoviak J, Stephenson LW, Edmunds LH Jr, et al. 1980. The intraaortic baloon pump: an analysis of five years' experience. Ann Thorac Surg. 29(5):451-8.

Mehta DP, Yoder EL, Appel J, et al. 1991. Heparin-induced thrombocytopenia and thrombosis: reversal with streptokinase a case report and review of literature. Am J Hematol. 36: 275-9.

Miranda M, Hossne NA, de Vilela AT, et al. 2014. Paraplegia After OffPump Coronary Artery Bypass Grafting. The Annals of Thoracic Surgery. 97(1), 326-7.

Mirhosseini SM, Meghdadi S, Moghaddam AS. 2017. Anterior Spinal Artery Syndrome Following Coronary Artery Bypass Grafting: a Case Report. Braz J Cardiovasc Surg. 32(2):136-7.

Müller KI, Steffensen LH, Johnsen SH. 2012. Thrombolysis in anterior spinal artery syndrome. BMJ Case Rep. Sep 7. bcr2012006862.

Pouplard C, May MA, Iochmann S, et al. 1999. Antibodies to platelet factor 4-heparin after cardiopulmonary bypass in patients anticoagulated with unfractionated heparin or a low-molecular-weight heparin: clinical implications for heparin-induced thrombocytopenia. Circulation. 99(19):2530-6.

Sabzi F, Faraji R. 2015. Double incontinence as a first symptom of saddle embolism of the aorta leads to sudden paraplegia. Arch Iran Med. 18(11): 796-8.

Scherr K, Urquhart G, Eichorst C, et al. 2006. Paraplegia after coronary artery bypass graft surgery: case report of a rare event. Crit Care Nurse. 26(5): 34-6, 38-40, 42-5.

Subramaniam A, Pick A, Tiruvoipati R. 2017. Anterior spinal artery syndrome after double valve replacement and coronary artery bypass surgery. Clin Case Rep. 5(5):714-17.

Taterra D, Skinning Srud B, Pękala PA, et al. 2019. Reply by Tomaszewski et al. to the letter by Jiang et al. regarding "Artery of Adamkiewicz: a meta-analysis of anatomical characteristics." Neuroradiology. 61(8):851-2.

Thomas NJ, Harvey AT. 1999. Paraplegia after coronary bypass operations: relationship to severe hypertension and vascular disease. J Thorac Cardiovasc Surg. Apr;117(4):834-6.

Tyras DH, Willman VL. 1978. Paraplegia following intraaortic balloon assistance. Ann Thorac Surg. 25(2):164-6.

White JM, Ruygrok PN. 2015. Intra-Aortic Balloon Counterpulsation in Contemporary Practice - Where Are We? Heart, Lung and Circulation. 24(4), 335-41. 